\title{
III-nitride blue and ultraviolet photonic crystal light emitting diodes
}

\author{
T. N. Oder, ${ }^{\text {a) }}$ K. H. Kim, J. Y. Lin, and H. X. Jiang ${ }^{\text {b) }}$ \\ Department of Physics, Kansas State University, Manhattan, Kansas 66506-2601
}

(Received 9 September 2003; accepted 29 November 2003)

\begin{abstract}
We present results on enhancement of $460 \mathrm{~nm}$ blue and $340 \mathrm{~nm}$ UV optical power output in III-nitride light emitting diodes (LEDs) using photonic crystals (PCs) under current injection. Triangular arrays of the PCs with diameter/periodicity of $300 / 700 \mathrm{~nm}$ were patterned using electron-beam lithography and inductively coupled plasma dry etching. The total power at $20 \mathrm{~mA}$ of $300 \times 300 \mu \mathrm{m}^{2}$ unpackaged LED chips revealed an increase by $63 \%$ and $95 \%$ for the blue and UV LEDs, respectively, as a result of the PC formation. Possible ways for further improving enhancement of light extraction using PCs are discussed. (c) 2004 American Institute of Physics.
\end{abstract}

[DOI: $10.1063 / 1.1644050]$

Solid-state light emitting diodes (LEDs) with high external efficiency are currently in high demand for a variety of applications including flat panel displays, printers, optical interconnects in computers, and general lighting. High efficient UV emitters are particularly sought for applications including chemical and biological agent detection and medical uses. However, while the internal quantum efficiency $(\mathrm{QE})$ of visible LEDs is close to $100 \%$; most of the light is lost to guided modes in the semiconductor materials and only about $1 /\left(4 n^{2}\right)$ of the light emitted radiates through the top and bottom. ${ }^{1}$ The need for improvement of extraction efficiency in LEDs is exceptionally great, especially for deep UV LEDs $(\lambda<340 \mathrm{~nm})$ based on III-nitride wide band gap semiconductors, which presently have very low QE. Much effort has been exerted in improving LED QE including the use of photon recycling schemes and novel geometrical designs aimed at enlarging escape cones of emitted light. ${ }^{2-4}$ Our group has also previously developed interconnected microdisk LED architecture as a method of enhancing extraction efficiency. ${ }^{5}$

The enhancement of external efficiency of LEDs using photonic crystals (PCs) has been studied mainly in the IR wavelength regions. These have been carried out using optical pumping on semiconductor materials not yet fabricated into electrically pumped LED devices and therefore lacked electrical data, which are essential in characterizing LEDs. ${ }^{6-9}$ Multiple scattering of photons by lattices of periodically varying refractive indices in PCs acts to form photonic band gaps (PBGs) in which propagation of certain wavelengths of the electromagnetic waves are prohibited. Ideal PBG is achieved by periodicity in three dimensions but for extraction of light in LEDs, it is sufficient to eliminate light propagation only in the horizontal plane with the use of two-dimensional (2D) PCs. For this purpose, triangular arrays of holes are typically etched into the semiconductor materials. Very little work in PCs involving the III-nitride materials in the blue/green and UV wavelengths has been reported due in part to the difficulty in fabrication associated

\footnotetext{
${ }^{a}$ Present address: Department of Physics and Astronomy, Youngstown Sate University, Youngstown, OH 44555.

${ }^{b)}$ Electronic mail: jiang@phys.ksu.edu
}

with the required nanometer scale periodicity. We recently reported a 20 -fold enhancement of light extraction using optical pumping in the nitride materials at $475 \mathrm{~nm} .{ }^{10}$ In this letter, we report the enhancement of extraction efficiency using PCs in III-nitride blue $(460 \mathrm{~nm})$ and UV (340 nm) LEDs under current injection. The optical output power at $20 \mathrm{~mA}$ of $300 \times 300 \mu \mathrm{m}^{2}$ unpackaged LED chips revealed an increase by $63 \%$ and $95 \%$ for the blue and UV LEDs, respectively, as a result of the PC formation. Our results mark a significant advance in the utilization of PCs for light extraction in nitride LEDs under current injection.

The III-nitride materials used were grown by metalorganic chemical vapor deposition (MOCVD) on sapphire substrates. Figures 1(a) and 1(b) shows the schematic diagrams
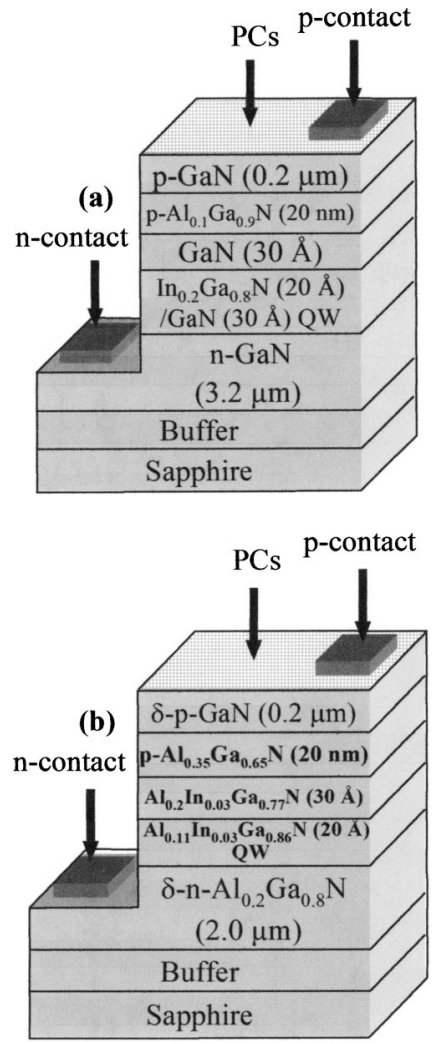

FIG. 1. Schematic diagrams of (a) $460 \mathrm{~nm}$ blue and (b) $340 \mathrm{~nm}$ UV LED structures. 

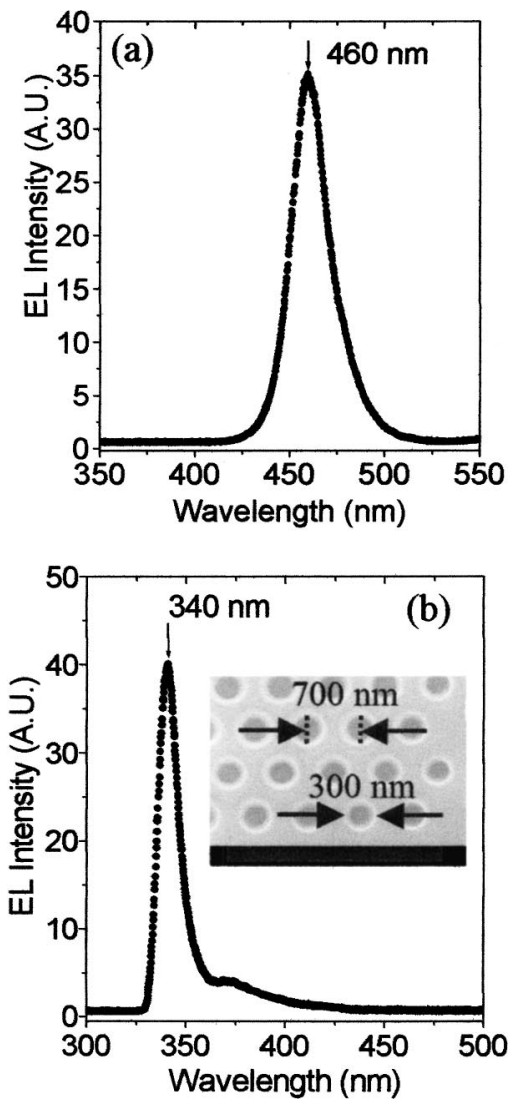

FIG. 2. (a) and (b) Typical EL data from $460 \mathrm{~nm}$ blue and $340 \mathrm{~nm}$ UV LEDs, respectively. The inset in (b) shows the SEM image of the nitride PCs after the triangular array of holes were etched in the nitride LEDs.

of our $460 \mathrm{~nm}$ blue and $340 \mathrm{~nm}$ UV LED structures, respectively. The active region for the blue LEDs was an $\mathrm{In}_{0.2} \mathrm{Ga}_{0.8} \mathrm{~N} / \mathrm{GaN}$ single quantum well (SQW) while for UV LEDs this was an $\mathrm{Al}_{0.11} \mathrm{In}_{0.03} \mathrm{Ga}_{0.86} \mathrm{~N} / \mathrm{Al}_{0.2} \mathrm{In}_{0.03} \mathrm{Ga}_{0.77} \mathrm{~N}$ SQW. Out of these materials, conventional broad area LEDs of $300 \times 300 \mu \mathrm{m}^{2}$ were fabricated by standard photolithography and inductively coupled plasma (ICP) dry etching. Additional patterns consisting of $200 \times 200 \mu \mathrm{m}^{2}$ square and ring-shaped LEDs of inner/outer diameters of $80 / 140$ and 90/180 $\mu \mathrm{m}$ were fabricated from the UV materials. Further details of the fabrication procedures are described elsewhere. ${ }^{11} I-V, L-I$, and electroluminescence (EL) measurements on specific LEDs were taken before and after the fabrication of PCs for comparison. The PCs with triangular lattice patterns of circular holes with diameter/periodicity of $300 / 700 \mathrm{~nm}$ were fabricated using electron-beam (e-beam) lithography and ICP dry etching as described in more detail elsewhere. ${ }^{10}$ Figures 2(a) and 2(b) shows the typical EL data for the $460 \mathrm{~nm}$ blue and $340 \mathrm{~nm}$ UV LEDs, respectively. The EL spectral shape (peak position and linewidth) is not altered by the formation of PCs. The inset in Fig. 2(b) shows the scanning electron microscopy (SEM) image of the nitride PCs after the triangular array of holes were etched in the nitride LEDs. Atomic force microscopy scan (data not shown) of the PCs was performed and showed that the etched depth was $200-250 \mathrm{~nm}$, indicating penetration through the $p$-type layers of the LED materials.

In Fig. 3(a), optical microscopy images of $300 \times 300 \mu \mathrm{m}^{2}$ blue LED without (left) and with (right) PCs are shown, both under $1 \mathrm{~mA}$ electrical current injection. The Downloaded 30 Mar 2011 to 129.118.237.235. Redistribution subject to AIP (a)
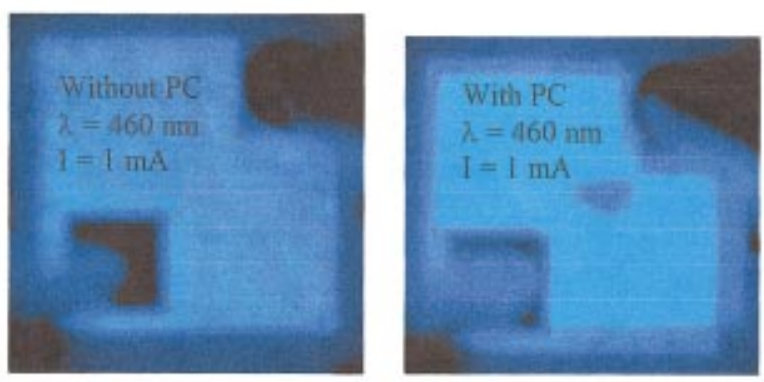

(b)
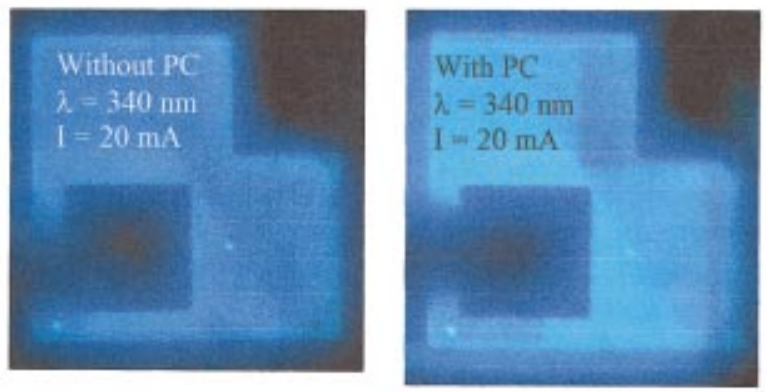

(c)
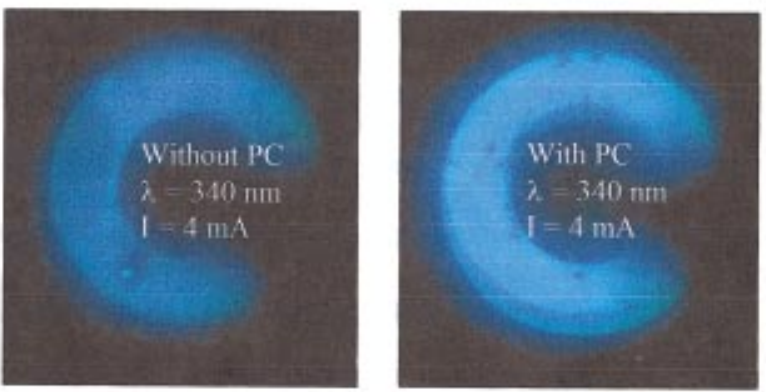

FIG. 3. (Color) (a) Optical microscopy images of $300 \times 300 \mu \mathrm{m}^{2}$ blue LED without (left) and with (right) PCs, both under $1 \mathrm{~mA}$ electrical current injection. (b) and (c) show optical microscopy images of $340 \mathrm{~nm}$ UV square and ring LEDs, respectively. The ring-LEDs have inner/outer diameters of $80 / 140 \mu \mathrm{m}$.

images were taken under the same camera settings for accurate comparison. The middle part in the right picture of Fig. 3(a) with less brightness corresponds to a damaged area resulting from alignment error during e-beam writing of the PCs. Figures 3(b) and 3(c), respectively, shows optical microscopy images of $340 \mathrm{~nm}$ UV square and ring LEDs. The ring-LEDs have inner/outer diameters of $80 / 140 \mu \mathrm{m}$. The LEDs patterned with PCs become much brighter under the same current injection. The $I-V$ and $L-I$ characteristics for square $\left(300 \times 300 \mu \mathrm{m}^{2}\right)$ blue LEDs are shown in Figs. 4(a) and 4(b), while those for UV LEDs are shown in Figs. 4(c) and $4(\mathrm{~d})$. The forward voltage $\left(V_{F}\right)$ shows an increase from 4.0 to $4.2 \mathrm{~V}$ for blue LEDs [Fig. 4(a)] while that for UV LEDs shows decrease by a much larger value from 7.6 to 6.0 $\mathrm{V}$ [Fig. 4(c)] following patterning of PCs. The slight increase in $V_{F}$ for blue LEDs is attributable to reduction in total active area and $p$-type contact region. The reason for the much larger drop in $V_{F}$ for UV LEDs is unclear but may be related to the enhanced current density as well as improved current spreading as a result of fabrication of the PCs. The optical power shown in Figs. 4(b) and 4(d) was obtained by averaging the values measured from the top and bottom sides of the unpackaged LED chips. At $20 \mathrm{~mA}$, the optical power of the $460 \mathrm{~nm}$ blue LED increased from 1.37 to $2.24 \mathrm{~mW}$ as a result of PC formation corresponding to an increase in optilicense or copyright; see http://apl.aip.org/about/rights_and_permissions 

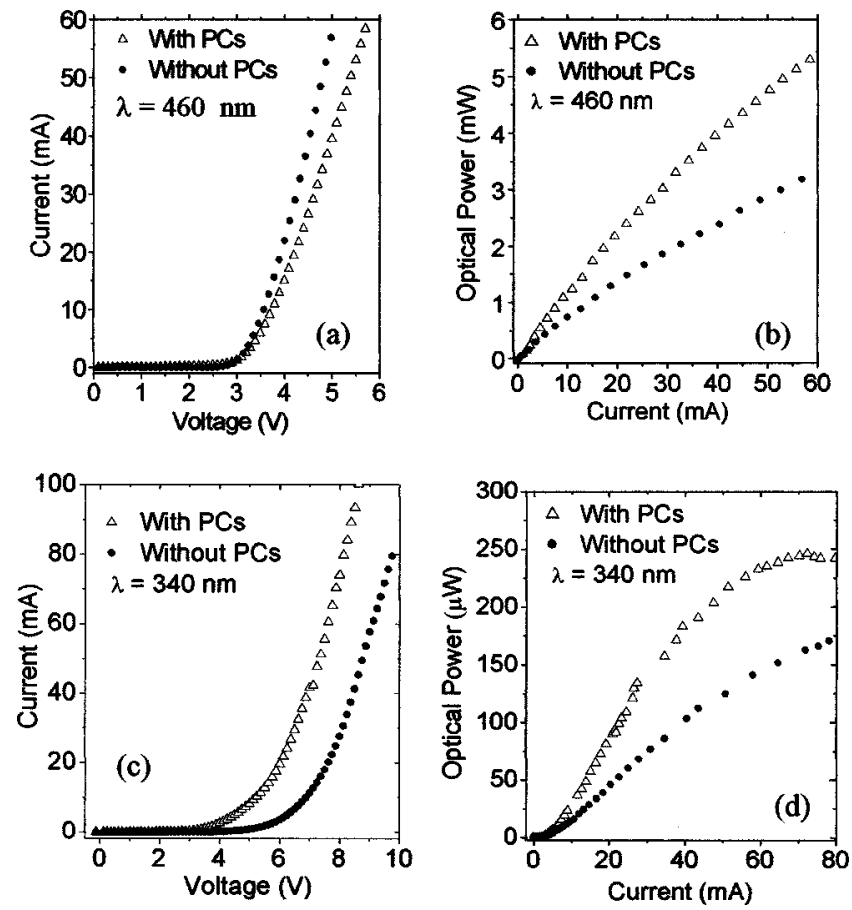

FIG. 4. The $I-V$ and $L-I$ characteristics of square $\left(300 \times 300 \mu \mathrm{m}^{2}\right)$ LEDs. (a) and (b) are results for $460 \mathrm{~nm}$ blue LEDs, while (c) and (d) are results for $340 \mathrm{~nm}$ UV LEDs. The optical power shown in (b) and (d) is obtained by averaging the values measured from the top and bottom side of the unpackaged bare LED chips. For $460 \mathrm{~nm}$ blue LEDs, the forward voltage $\left(V_{F}\right)$ shows an increase by about $0.2 \mathrm{~V}$ and the optical power enhanced by $63 \%$ at $20 \mathrm{~mA}$ following patterning of PCs. For $340 \mathrm{~nm}$ UV LEDs, The forward voltage $\left(V_{F}\right)$ shows a decrease by about $1.6 \mathrm{~V}$ following patterning of PCs while the power shows an enhancement by $95 \%$ at $20 \mathrm{~mA}$. While the optical power level is still low for the UV LEDs studied here, comparison is being made on the overall intensity enhancement using PCs. Most recently, our MOCVD growth endeavors have since yielded UV LED materials with improved qualities and higher optical power output (exceeding $1 \mathrm{~mW}$ at 120 $\mathrm{mA} \mathrm{dc}$ at $300 \mathrm{~nm}$ ) (see Ref. 12).

cal power by $63 \%$. For the $340 \mathrm{~nm}$ UV LEDs, the optical power increased from 44.5 to $86.6 \mu \mathrm{W}$, corresponding to about 95\% increase in intensity. It should be pointed out that while the optical power level is still low for the UV LEDs studied here, comparison is being made on the overall intensity enhancement using PCs. Most recently, our MOCVD growth endeavors have since yielded UV LED materials with improved qualities and higher optical power output (exceeding $1 \mathrm{~mW}$ at $120 \mathrm{~mA} \mathrm{dc}$ at $300 \mathrm{~nm}) .^{12}$

Enhancement of light extraction using 2D PCs is achieved in two possible ways. First, if the lattice dimensions of the PCs are tuned correctly $(\sim \lambda / 2), \mathrm{PBG}$ is created as a result of multiple scattering of photons by lattices of periodically varying refractive indices. Light generated in the band gap region can only radiate outward since lateral propagation of guided modes are prohibited in the band gap, thus improving extraction efficiency. ${ }^{13,14}$ The second possibility is due to the fact that refractive index periodicity creates a cutoff frequency for the guided modes. Modes above the cutoff frequency are leaky and couple to free space, again improving output efficiency. ${ }^{6,7}$ The PCs offer a short length where light escapes before being dissipated by parasitic absorption. In contrast, random scattering from a randomly textured surface may improve LED output efficiency but is subject to parasitic absorption.

PBG is normally characterized by its midgap ratio Downloaded 30 Mar 2011 to 129.118 .237 .235 . Redistribution subject to
$\left(\Delta \omega / \omega_{0}\right)$ where $\Delta \omega$ is the frequency range spread out by the PBG and $\omega_{0}$ is the frequency at the middle of the gap (midgap). The midgap ratio is typically small, of the order of $20 \%$. The midgap frequency for triangular air columns in $\mathrm{GaN}$ with dielectric constant $\varepsilon \approx 8.9$ is estimated to be at a normalized frequency $(a / \lambda=\omega a / 2 \pi c)$ of $0.5 .^{9,14}$ The normalized frequency corresponding to our PCs structure ( $a$ $=700 \mathrm{~nm}$ ) is about $1.5 / 2.1$ for the $460 / 340 \mathrm{~nm}$ wavelengths. This implies that photon modes in our structure lie above the cutoff frequency of the guided modes. Therefore, enhancement in our LEDs is mainly due to coupling to leaky modes as discussed earlier. We believe that further enhancement in extraction efficiency in III-nitride LEDs using PCs will be realized with additional improvement in the design and processing of the LEDs particularly in the following areas: (a) increasing etch depth to ensure penetration into the active layers, (b) fabricating PCs with reduced diameter/periodicity to target the midgap region of the PBG, and (c) minimizing the loss of charged carriers which occurs in etching-induced defects by separating the current injection region from light extraction region, and (d) design of LED configuration to take advantages of PCs and current spreading.

In summary, we have achieved a power enhancement by $63 \%$ and $95 \%$ for blue $(460 \mathrm{~nm})$ and UV (340 nm) light extraction in III-nitride LEDs under current injection using PCs. Triangular lattice PCs with diameter/periodicity of 300/ $700 \mathrm{~nm}$ were patterned using electron-beam lithography and ICP dry etching. Our results shows significant achievement in the use of PCs to realize enhanced power output in fabricated LEDs.

The authors wish to acknowledge support by grants from DARPA, ARO, DOE, and NSF.

${ }^{1}$ M. Boroditsky and E. Yablonovitch, Proc. SPIE 3002, 119 (1997).

${ }^{2}$ W. N. Carr and G. E. Pittman, Appl. Phys. Lett. 3, 173 (1963).

${ }^{3}$ I. Schnitzer, E. Yablonovitch, C. Caneau, and T. J. Gmitter, Appl. Phys. Lett. 62, 131 (1993).

${ }^{4}$ I. Schnitzer, E. Yablonovitch, C. Caneau, T. J. Gmitter, and A. Scherer, Appl. Phys. Lett. 63, 2174 (1993).

${ }^{5}$ S. X. Jin, J. Li, J. Y. Lin, and H. X. Jiang, Appl. Phys. Lett. 77, 3236 (2000).

${ }^{6}$ A. A. Erchak, D. J. Ripin, S. H. Fan, P. Rakich, J. D. Joannoupoulos, E. P. Ippen, G. S. Petrich, and L. A. Kolodziejski, Appl. Phys. Lett. 78, 563 (2001).

${ }^{7}$ H. Y. Ryu, J. K. Hwang, Y. J. Lee, and Y. H. Lee, IEEE J. Sel. Top. Quantum Electron. 8, 321 (2002).

${ }^{8}$ M. Boroditsky, T. F. Krauss, R. Coccioli, R. Vrijen, R. Bhat, and E. Yablonovitch, Appl. Phys. Lett. 75, 1036 (1999).

${ }^{9}$ Y. J. Lee, S. H. Kim, J. Huh, G. H. Kim, Y. H. Lee, S. H. Cho, Y. C. Kim, and Y. R. Do, Appl. Phys. Lett. 82, 3779 (2003).

${ }^{10}$ T. N. Oder, J. Shakya, J. Y. Lin, and H. X. Jiang, Appl. Phys. Lett. 83, 1231 (2003).

${ }^{11}$ K. H. Kim, J. Li, S. X. Jin, J. Y. Lin, and H. X. Jiang, Appl. Phys. Lett. 83, 566 (2003).

${ }^{12}$ K. H. Kim, Z. Y. Fan, M. L. Nakarmi, M. Khizar, S. X. Jin, J. Y. Lin, and H. X. Jiang (unpublished).

${ }^{13}$ E. Yablonovitch, J. Opt. Soc. Am. B 10, 283 (1993).

${ }^{14}$ J. D. Joannopoulos, R. D. Meade, and J. N. Winn, Photonic Crystals, Molding the Flow of Light (Princeton University Press, Princeton, 1995). 